\title{
Microdiscectomia lombar: acessos interlaminar e intertransverso com uso de afastador de Caspar tubular cilíndrico e dreno de Penrose como auxiliar no afastamento da musculatura - estudo de 502 casos
}

\section{Lumbar Microdiscectomy: Interlaminar and Intertransverso Access using Tubular Retractor Caspar Cylindrical and Penrose Drain as an Aid in Muscular Retraction - Study of 502 Cases}

José Calasans dos Santos ${ }^{1}$ Camila Ricci Calasans ${ }^{2}$ Victor Gama Mascarenhas ${ }^{2}$

${ }^{1}$ Membro titular da Sociedade e da Academia Brasileira de Neurocirurgia. Membro Efetivo da Sociedade Brasileira de Coluna. Chefe do serviço de Neurocirurgia do Hospital da Unimed, Aracaju, Sergipe, Brasil

${ }^{2}$ Acadêmico de Medicina da Faculdade de Tecnologia e Ciências,

Salvador, Bahia, Brasil

Arq Bras Neurocir 2015;34:185-194.
Address for correspondence José Calasans dos Santos, MR, Av. Beira Mar, 1152, apto. 1404, bairro Treze de Julho, Aracaju, SE, Brasil. CEP 49020-010 (e-mail: j_calasans@hotmail.com).

\section{Resumo \\ Palavras-Chave \\ - hérnia discal lombar \\ - microdiscectomia \\ - acesso interlaminar \\ - acesso intertransverso}

Abstract
$\begin{aligned} & \text { Keywords } \\ & \text { - lumbar disc hernia } \\ & \text { - microdiscectomy } \\ & \text { - interlaminar and } \\ & \text { intertransverse } \\ & \text { access }\end{aligned}$

Os autores fazem uma revisão da literatura abordando conhecimentos neuroanatômicos da raiz e do gânglio da raiz dorsal. Descrevem a técnica para os acessos interlaminar e intertransverso com o uso de afastador de Caspar tubular cilíndrico e dreno de Penrose como auxiliares no afastamento da musculatura. Basearam-se em 502 casos operados em 25 anos. O objetivo deste trabalho é descrever uma técnica com incisão pequena na pele, baixa agressividade para as estruturas anatômicas, sem perda funcional da musculatura paravertebral, campo cirúrgico amplo, facilidade de execução com as duas mãos, alta hospitalar precoce em torno de 24 horas e baixo custo.

The authors review the literature addressing neuro-anatomical knowledge of the root and root ganglion dorsal. Describe the technique for the interlaminar and intertransverso access using tubular retractor Caspar cylindrical Penrose drain as an aid in muscular retraction. Based-seem 502 cases operated in 25 years. The objective of this paper is to describe a technique with small skin incision, low aggressiveness anatomical structures without functional loss of paraspinal musculature, broad surgical field, easy work with both hands, high early hospital about 24 hours and low cost.
Recebido

August 25, 2014

Aprovado

March 31, 2015
DOI http://dx.doi.org/ 10.1055/s-0035-1554047. ISSN 0103-5355.
Copyright $\odot 2015$ by Thieme Publicações License terms

Ltda, Rio de Janeiro, Brazil c) $(\oplus) \$$ 


\section{Introdução}

As hérnias discais lombares (HDL) são relativamente frequentes e acometem de 2 a $3 \%$ da população, principalmente entre 40 e 50 anos, com discreta predominância para o sexo masculino, e são a principal causa de cirurgia de coluna do adulto. Schmorl foi o primeiro a descrever, em 1927, a ruptura de disco intervertebral no interior do corpo de uma das vértebras. ${ }^{1,2}$ Contudo, já em 1909, Krause descrevera uma operação realizada em determinado paciente no qual Oppenheim havia diagnosticado uma lesão localizada em L4. 2,3 Krause encontrou massa extradural descrita pelo anatomopatologista como sendo um condroma; a operação resultou em cura. ${ }^{3}$ Mixter e Barr demonstraram, em 1934, que aquelas lesões eram, na realidade, fragmentos de discos intervertebrais responsáveis por casos de ciática, o que permitiu avanços diagnósticos e terapêuticos. ${ }^{4}$ Naquela época, os recursos diagnósticos eram imprecisos, e as cirurgias, mutiladoras, consistiam em laminectomias com exploração de múltiplos níveis em busca da hérnia discal. A mortalidade era alta, em torno de $20 \% .^{5}$ Love, em 1939 , descreveu uma técnica para exérese do disco por meio da flavectomia (fenestração interlaminar do ligamento amarelo), associando-se, se necessário, hemilaminectomia, com avanço na técnica cirúrgica e diminuição da mortalidade. ${ }^{6}$ Em 1977, Caspar e Loew relataram uma técnica cirúrgica através de pequena incisão, com auxílio do microscópio cirúrgico e instrumental especializado, iniciando a era microcirúrgica para o tratamento das hérnias discais. ${ }^{1,7}$ Com o desenvolvimento da microcirurgia em geral, Willams, em 1978, desenvolveu uma técnica para a operação de hérnia de disco, com poucas modificações, a qual denominou microdiscectomia, sendo popularizada desde então. ${ }^{2,8}$

\section{Definição}

Hérnia discal é o deslocamento de qualquer volume de conteúdo discal para além dos limites do espaço do disco intervertebral, limitado no sentido craniocaudal pelas placas terminais e, circunferencialmente, pelas bordas dos corpos vertebrais. ${ }^{9}$ A herniação pode ser anterior, intravertebral, posteromediana, posterolateral, foraminal e extraforaminal. As anteriores e as intravertebrais (nódulos de Schmorl) constituem entidades de pouca relevância clínica. ${ }^{9}$

\section{Anatomia da raiz e do gânglio da raiz dorsal}

O gânglio da raiz dorsal (GRD), local em que os corpos das células do nervo sensorial estão fixados, é vulnerável à compressão por mudanças degenerativas estruturais do disco, faceta, pedículo, lâmina e ligamento amarelo. Ele modula a dor dos segmentos de movimento da coluna através de neuropeptídeos intrínsecos. O GRD e a raiz dorsal podem ser bifurcados ou não bifurcados. As raízes L4 e L5 se bifurcam e S1 não se bifurca. As posições do GRD podem ser classificadas em três tipos: intraespinal, intraforaminal e extraforaminal. Nas raízes nervosas L4 e L5, o GRD é, na maior parte das vezes, intraforaminal, enquanto em S1 é
Tabela 1 Localização do GRD de acordo com o nível da raiz

\begin{tabular}{|l|l|}
\hline Nível & Localização do GRD \\
\hline L1 & $92 \%$ no FIL \\
\hline L2 & $98 \%$ no FIL \\
\hline L3 & $100 \%$ no FIL \\
\hline L4 & $100 \%$ no FIL \\
\hline L5 & $98 \%$ no FIL \\
\hline S1 & $79 \%$ na região intraespinal \\
\hline
\end{tabular}

Abreviações: GRD, gânglio da raiz dorsal - local em que os corpos das células do nervo sensorial estão localizados; FIL, forame intervertebral lombar.

intraespinal. ${ }^{10} \mathrm{~A}$ - Tabela 1 demonstra a localização do GRD de acordo com o nível da raiz.

A raiz nervosa lombar ocupa de 23 a 30\% da área do forame intervertebral. ${ }^{10} \mathrm{~A}$ raiz nervosa e o GRD de S1 podem estar comprimidos por hérnia de disco ou por mudanças degenerativas da faceta de L5-S1, porque o GRD de S1 é o mais intraespinal de todos os complexos do nervo lombar. Mudanças no GRD produzem radiculopatia e hiperalgesia térmica. A raiz nervosa motora (ventral) e a raiz nervosa sensorial (dorsal) passam posterior e lateralmente ao disco intervertebral. As raízes nervosas dorsais apresentam diâmetro maior do que a raiz nervosa ventral e, dessa forma, maior suscetibilidade dos axônios a forças compressivas. As raízes nervosas da cauda equina possuem somente endoneuro, sendo desprovidas de epineuro e perineuro, ao contrário dos nervos periféricos e, portanto, mais um fator de suscetibilidade a forças compressivas. As raízes do nervo S1 têm aproximadamente $170 \mathrm{~mm}$ de comprimento, enquanto as raízes do nervo de $\mathrm{L} 1$ têm $60 \mathrm{~mm}$. As raízes nervosas devem mudar de comprimento, dependendo do grau de flexão, extensão, inclinação lateral e rotação. As raízes nervosas lombares limitadas em movimentos por fibrose ou compressão intraespinal ou extraespinal criam uma tração no complexo da raiz nervosa, o que leva a isquemia e disfunção neural secundária. ${ }^{10} \mathrm{Na}$ cirurgia de HDL a raiz deve apresentar uma boa mobilidade e, se isso não acontecer, o paciente não evoluirá com uma melhora satisfatória.

\section{Classificação}

As HDL podem ser classificadas quanto ao plano horizontal, à morfologia, à integridade do anel fibroso, à localização no interior do canal vertebral e ao volume no interior do canal vertebral (-Figs. 1-7).

Plano horizontal: cortes axiais na ressonância nuclear magnética (RNM) ${ }^{11}$

- Focal - quando menor que $25 \%$ da circunferência do disco

- Base larga - quando seu tamanho está entre 25 e 50\% da circunferência do disco

- Generalizada - maior que 50\% da circunferência do disco Morfologia: cortes axiais na $\mathrm{RNM}^{11}$ 


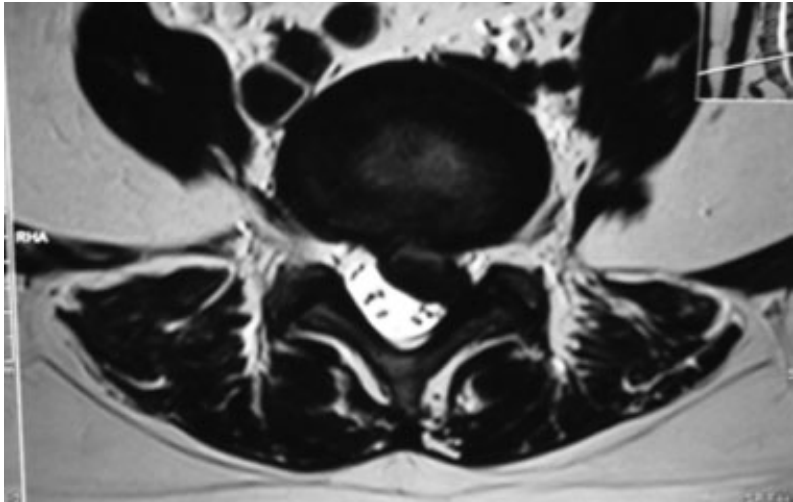

Fig. 1 Hérnia discal lombar intraespinal (posteromédio lateral) L4-L5.

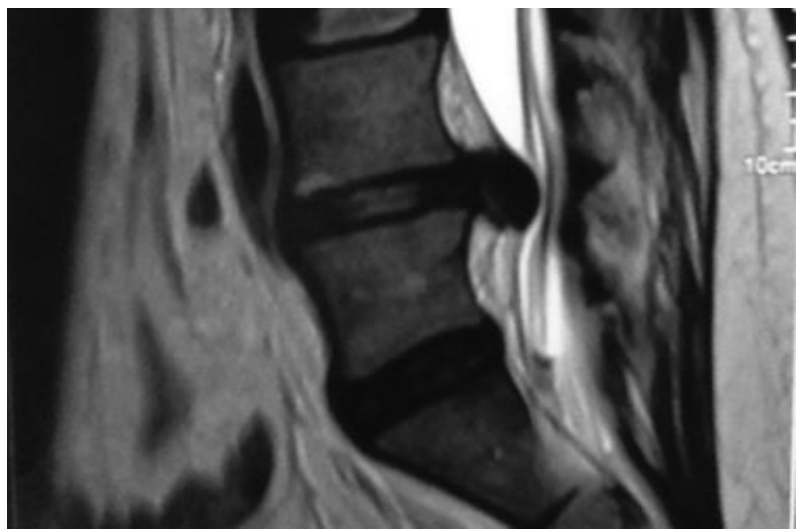

Fig. 2 Hérnia discal lombar não contida L4-L5.

- Protusa - base maior que altura

- Extrusa - altura maior que base

Integridade do anel fibroso: cortes axiais e sagitais na $\mathrm{RNM}^{11}$

- Contida - há integridade parcial do ânulo fibroso

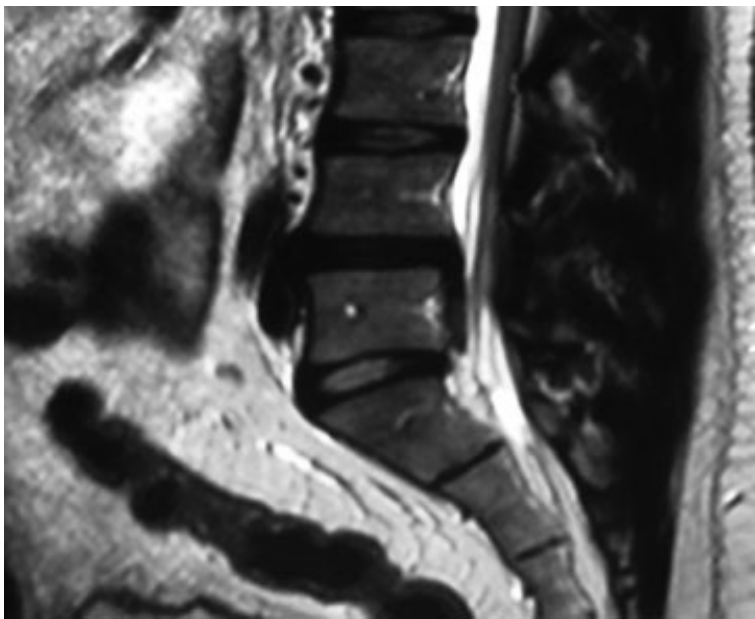

Fig. 3 Hérnia discal lombar migrada caudalmente sem sequestro L4-L5.

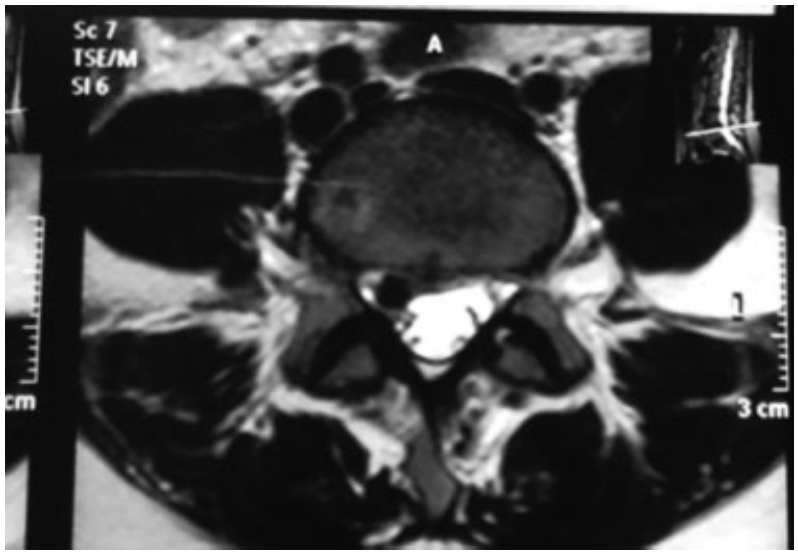

Fig. 4 Hérnia discal lombar posterolateral L4-L5.

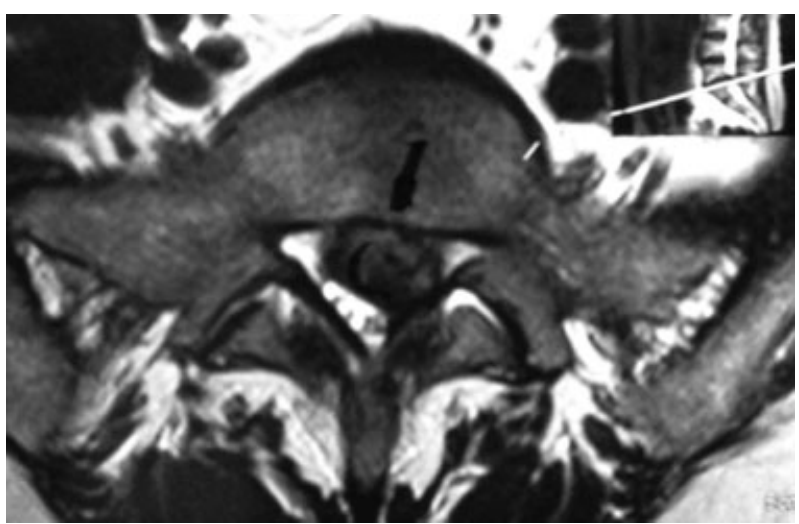

Fig. 5 Hérnia discal lombar extrusa e gigante L5-S1.

- Não contida - não há integridade; podem ser migradas cranial ou caudalmente com ou sem sequestro

Localização no interior do canal vertebral: cortes axiais e sagitais na $\mathrm{RNM}^{11}$

- Posterocentral ou posteromediana

- Posterolateral

- Foraminal

- Extraforaminal

Volume no interior do canal vertebral: cortes axiais na RNM $^{12}$

- Pequena - até $12 \%$ do canal vertebral

- Média - 12 a $25 \%$ do canal vertebral

- Grande - 25 a 50\% do canal vertebral

- Gigante - maior que 50\% do canal vertebral

\section{História natural}

Trata-se de uma doença benigna com característica evolutiva para a cura espontânea. Weber demonstrou que os resultados de pacientes não operados e operados em 4 anos são semelhantes e iguais em 10 anos, sendo que os operados 


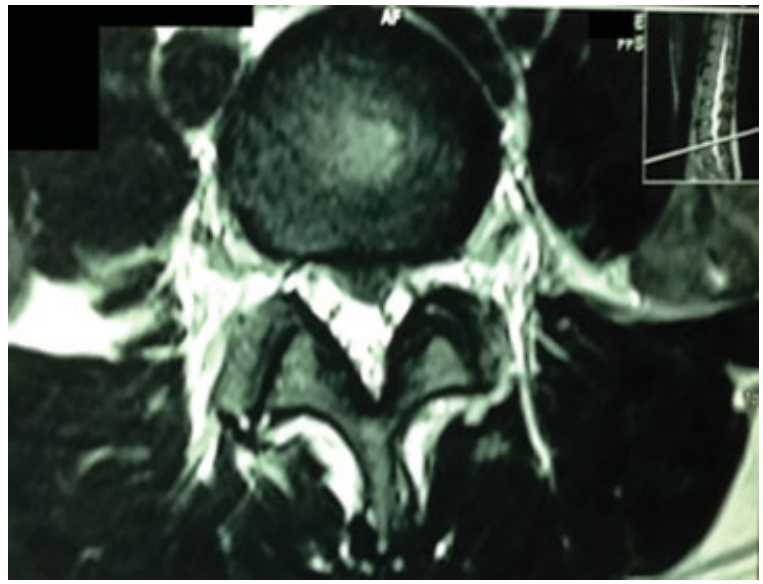

Fig. 6 Hérnia discal lombar protusa e mediana L4-L5.

obtiveram melhora significativa no primeiro ano pós-cirúrgico, comparados aos não operados. ${ }^{13} \mathrm{O}$ sintoma mais frequente da HDL é a ciatalgia, caracterizada por dor de padrão radicular irradiada para o membro inferior que alivia quando o paciente deita, e piora quando ele senta ou caminha. Aproximadamente 50\% dos pacientes acometidos recuperam-se no primeiro mês, 95\% em até 6 meses e apenas de 1 a $6 \%$ necessitam de cirurgia. As - Figs. 8 e 9 demonstram que a pressão no disco é maior quando a pessoa está sentada e diminui quando fica em ortostase, sendo menor ainda

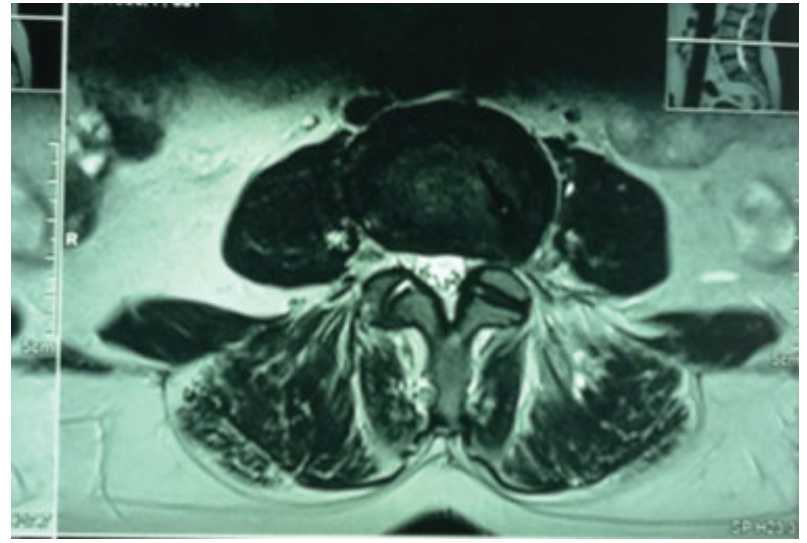

Fig. 7 Hérnia discal lombar extraespinal (extraforaminal) L3-L4.

quando o indivíduo está deitado ou assume o decúbito dorsal.

\section{Material e Métodos}

Os 502 pacientes portadores de hérnia discal lombar foram submetidos à microdiscectomia lombar ao longo de 25 anos (1988-2013). Para o acesso interlaminar foram usados os afastadores lombares Valin, Scoville, Markham-Meyerding, Williams, Taylor, Caspar, associação de Caspar e Taylor,

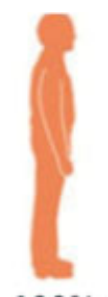

$100 \%$

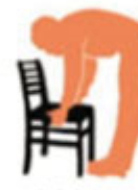

$200 \%$

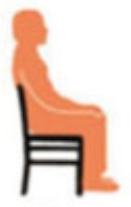

$150 \%$

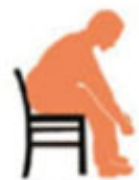

$250 \%$

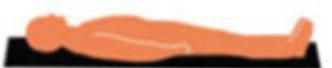

$25 \%$

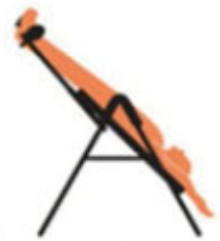

$0 \%$

Fig. 8 Pressão sobre o disco de acordo com a posição do corpo. Fonte: http://www.neurocirurgiabh.com
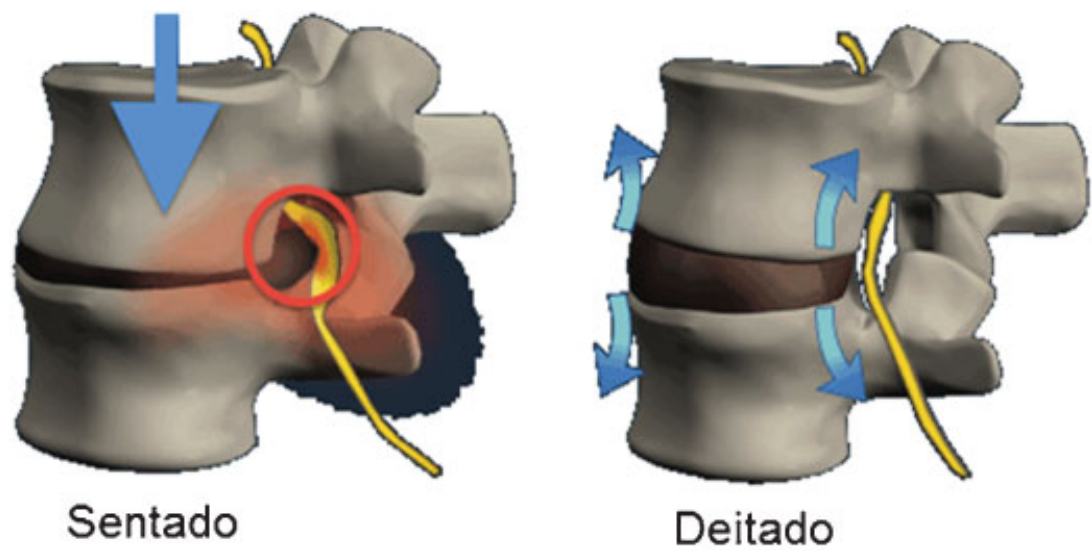

Fig. 9 Pressão sobre o disco de acordo com a posição. Fonte: http://www.neurocirurgiabh.com 
Caspar e Williams, Caspar associado a um dreno de Penrose (dreno laminar confeccionado de látex de $30 \mathrm{~cm}$ de comprimento e $3 \mathrm{~cm}$ de largura) como afastador da musculatura lateral e tubos dilatadores. Já para o acesso intertransverso, usamos o Caspar com dois drenos de Penrose como afastadores da musculatura medial e lateral, e os mesmos afastadores do acesso interlaminar, exceto o Taylor, usado somente para o acesso interlaminar. Foi realizada comparação quanto aos afastamentos cranial, caudal, lateral e medial, quanto à ampliação do campo cirúrgico com pequena incisão na pele e quanto à necessidade de abordar dois ou três espaços discais vizinhos.

\section{Diagnóstico}

É realizado através da anamnese, exame físico, exame neurológico e exames complementares. $\mathrm{O}$ exame complementar inicial é o estudo radiológico simples em quatro posições: anteroposterior, perfil, oblíquo anterior direito e oblíquo anterior esquerdo. Os autores realizam - em todos os pacientes que serão submetidos à cirurgia - radiografias simples em ortostase, incluindo as cabeças femorais no perfil para ter a incidência pélvica e acrescentando o perfil em flexão e extensão para observar a existência de listeses.

A RNM da coluna lombossacra sem contraste é o padrãoouro no diagnóstico radiológico das hérnias discais. Caso sejam necessárias, a tomografia computadorizada, tomomielografia e raramente a eletroneuromiografia podem ser realizadas.

Também é preciso afastar as causas mecânicas, degenerativas, infecciosas, tumorais, traumáticas, reumáticas, viscerais e psicogênicas.

\section{Tratamento}

\section{Tratamento Conservador}

Objetiva o alívio da dor, a preservação da função e o retorno precoce às atividades habituais. 0 tratamento cirúrgico desnecessário deve ser evitado. O resultado do tratamento conservador a médio e longo prazo tende a ser igual ao resultado do tratamento cirúrgico, entretanto, em pacientes muito limitados pela dor, este último produz alívio quase imediato dos sintomas. O tratamento conservador por 6 semanas tem bons resultados em até 80 a $90 \%$ dos casos. ${ }^{14}$ A imensa maioria das HDL evolui para a cura sem qualquer relação com a melhora radiológica, ou seja, nunca se deve tratar a imagem da hérnia, mas sim os seus sinais e sintomas. No início, o repouso está indicado, porém não deve ser absoluto. A própria dor obriga o paciente a um repouso relativo que não deve exceder a primeira semana da ciatalgia.

O tratamento medicamentoso inclui analgésicos, antiinflamatórios e miorrelaxantes. É importante que uma analgesia eficiente logo no início do tratamento seja obtida. Além de analgésicos e anti-inflamatórios não hormonais (AINH) em dose plena, os corticosteroides em doses regressivas por curto período podem ser utilizados. O uso de corticosteroides por via epidural permite maior concentração de corticoide local, minimizando os efeitos sistêmicos indesejáveis.
Sua utilização pode levar a um alívio sintomático mais rápido, muito embora não tenha papel na evolução final do quadro; também não devemos esquecer da possibilidade de hematomas e abscessos epidurais relacionados com as infiltrações locais prévias. Na fase inicial da doença, deve-se evitar qualquer tipo de manipulação, e o papel da fisioterapia limita-se às medidas analgésicas e de relaxamento muscular. Com a melhora dos sintomas, a reabilitação deve ser progressivamente indicada, para que novas crises sejam evitadas, através de exercícios de alongamento e de fortalecimento muscular.

\section{Tratamento Cirúrgico}

Atualmente a síndrome da cauda equina é a única indicação absoluta para tratamento cirúrgico de urgência. ${ }^{9,15}$ Na nossa casuística, operamos 4 casos $(0,79 \%)$ de síndrome da cauda equina. As indicações mais comuns da HDL são relativas, sendo a falha do tratamento conservador o motivo mais frequente para o tratamento cirúrgico. A cirurgia pode ser indicada se a ciatalgia é intensa ou limitante, se os sinais de sofrimento radicular estão presentes e se os sintomas persistem por mais de 6 semanas. Alguns casos evoluem de forma hiperálgica persistente e podem ter indicação cirúrgica mais precoce, que também é recomendada em casos de progressão de déficit motor. 0 sucesso do tratamento cirúrgico depende da consistência e da concordância dos achados clínicos com os exames complementares.

Um dos instrumentos mais importantes para a realização dos acessos descritos a seguir é o afastador de Caspar. Foram utilizados dois afastadores de Caspar, sendo um de $6 \mathrm{~cm}$ de profundidade e outro de $8 \mathrm{~cm}$ de profundidade; este último, por sua maior profundidade, é utilizado preferencialmente nos pacientes obesos. As tabelas a seguir revelam a abertura do Caspar de acordo com sua expansão (-Tabelas 2 e $\mathbf{3}$ ).

As HDL podem ser operadas por quatro acessos: interlaminar, intertransverso, lateral e anterior. Neste trabalho serão abordados os acessos interlaminar e intertransverso.

\section{Acesso Interlaminar}

Neste acesso, operam-se as HDL posterocentrais, laterais e foraminais, ou seja, as hérnias intraespinhais. Na nossa casuística, operamos 490 hérnias intraespinhais (97,6\%). A posição cirúrgica é a Tarlov ( - Fig. 10) tipo genopeitoral que permite uma boa flexão da coluna lombar, flacidez abdominal e redução da pressão abdominal com menor congestão venosa, facilitando a cirurgia. Nas pernas utilizam-se meias elásticas para evitar trombose. Deve-se ter cuidado especial com os olhos para evitar compressão ocular.

Tabela 2 Abertura do Caspar sem expansão ou fechado

\begin{tabular}{|l|l|l|}
\hline & Caspar 1 & Caspar 2 \\
\hline ASMi & $2,5 \mathrm{~cm}$ & $1,5 \mathrm{~cm}$ \\
\hline AIMi & $1,5 \mathrm{~cm}$ & $1,0 \mathrm{~cm}$ \\
\hline
\end{tabular}

Abreviações: ASMi, abertura superior mínima; AIMi, abertura inferior mínima. 
Tabela 3 Abertura do Caspar com expansão ou aberto

\begin{tabular}{|l|l|l|}
\hline & Caspar 1 & Caspar 2 \\
\hline ASMa & $4,0 \mathrm{~cm}$ & $6,0 \mathrm{~cm}$ \\
\hline AIMa & $6,0 \mathrm{~cm}$ & $8,0 \mathrm{~cm}$ \\
\hline
\end{tabular}

Abreviações: ASMa, abertura superior máxima; AIMa, abertura inferior máxima.

Após posicionamento adequado do paciente, localiza-se o espaço discal a ser abordado com auxílio de intensificador de imagem em visão lateral. Coloca-se a pinça Kelly reta na linha espinhal (média) da pele em direção ao disco herniado, sendo que este é o ponto central da incisão cirúrgica (-Fig. 11). Após antissepsia e assepsia, faz-se uma incisão mediana de 2 a 2,5 cm na pele. Realiza-se uma incisão em meia lua da fáscia toracolombar homolateral à hérnia, sendo que a base fica em contato com a linha média, e rebate-se medialmente com ancoragem com fio de náilon de $2 \mathrm{~mm}$. Com auxílio do bipolar é realizada uma pequena coagulação entre o ligamento supraespinal e a musculatura paraespinal para facilitar a dissecção subperiostal da musculatura das lâminas das vértebras cranial e caudal e de todo o recesso lateral. Antes de colocar o afastador de Caspar, palpa-se com o dedo indicador o espaço interlaminar para que se certifique a posição, pois o espaço L5-S1 é facilmente identificado por ser o último e pela presença da asa sacral. O L4 é também facilitado por sua vizinhança com o L5-S1. Já em espaços acima de L4-L5, mesmo com a marcação da pele, é prudente realizar outra imagem lateral com intensificador de imagem para confirmar o espaço discal. Certificado o espaço, coloca-se o afastador de Caspar que afasta de modo satisfatório a musculatura cranial e caudalmente, porém o recesso lateral é visualizado com maior facilidade quando se coloca um dreno de Penrose de $30 \mathrm{~cm}$ de comprimento e $3 \mathrm{~cm}$ de largura com auxílio de Kelly curva por dentro da musculatura lateral que fica entre as lâminas do Caspar (-Figs. 12 e 13). Ao tracionar o dreno lateralmente, há elevação e afastamento da musculatura lateral com excelente visão do recesso lateral. Coloca-se o microscópio, visualiza-se o ligamento amarelo e com cureta

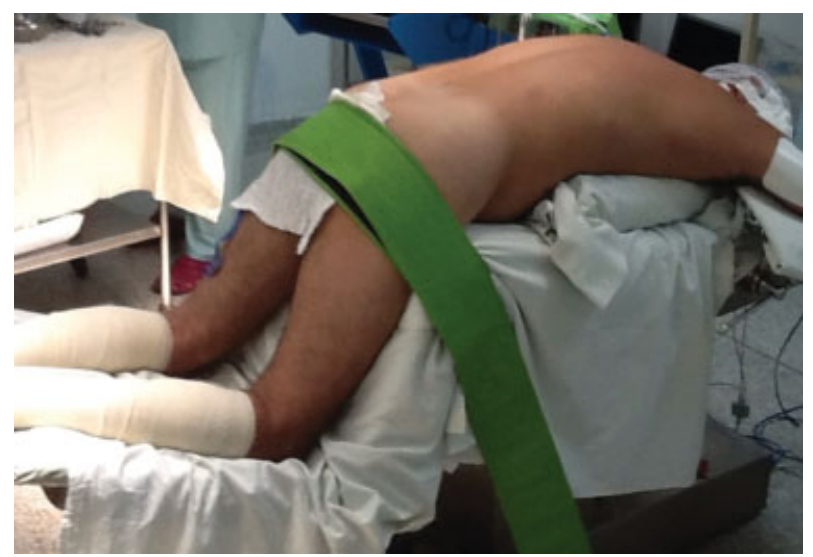

Fig. 10 Posição cirúrgica do acesso interlaminar.

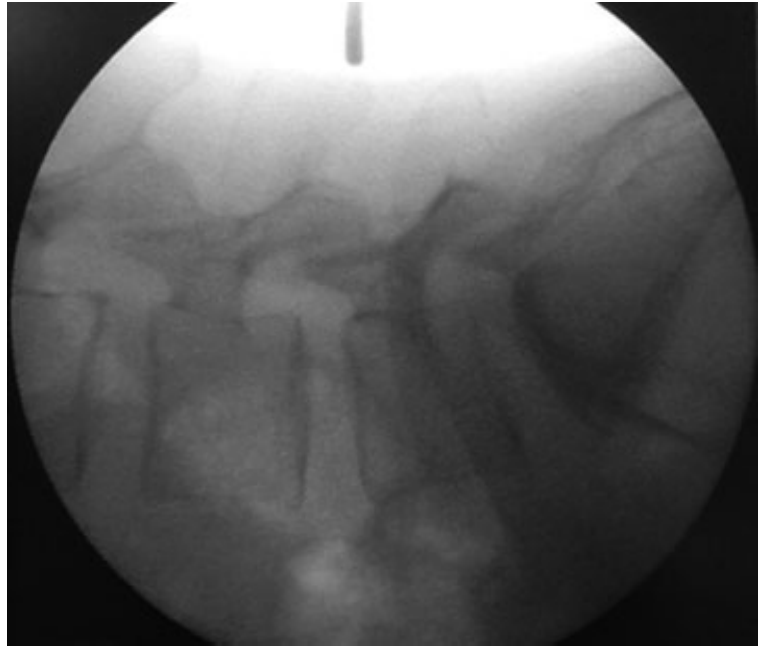

Fig. 11 Ponto central da incisão cirúrgica do acesso interlaminar para abordar o disco L4L5.

de $2 \mathrm{~mm}$ promove-se desinserção do ligamento amarelo da lâmina superior. Com broca de drill de alta rotação ou sacabocado tipo Kerrison, realiza-se um pequeno desbaste da lâmina superior (cranial) com visualização dos bordos livres do ligamento amarelo e do espaço epidural. Coloca-se cotonoide neste espaço para afastar o ligamento amarelo da gordura epidural, vasos epidurais, saco dural e raiz. 0 mesmo procedimento é feito com o ligamento amarelo e lâmina inferior (caudal), e coloca-se outro cotonoide no espaço epidural. É feito um pequeno desbaste com drill ou Kerrison do recesso lateral para melhor visualização da parte mais lateral do ligamento amarelo. Como o ligamento amarelo já está afastado por cotonoides das estruturas neurais, utiliza-

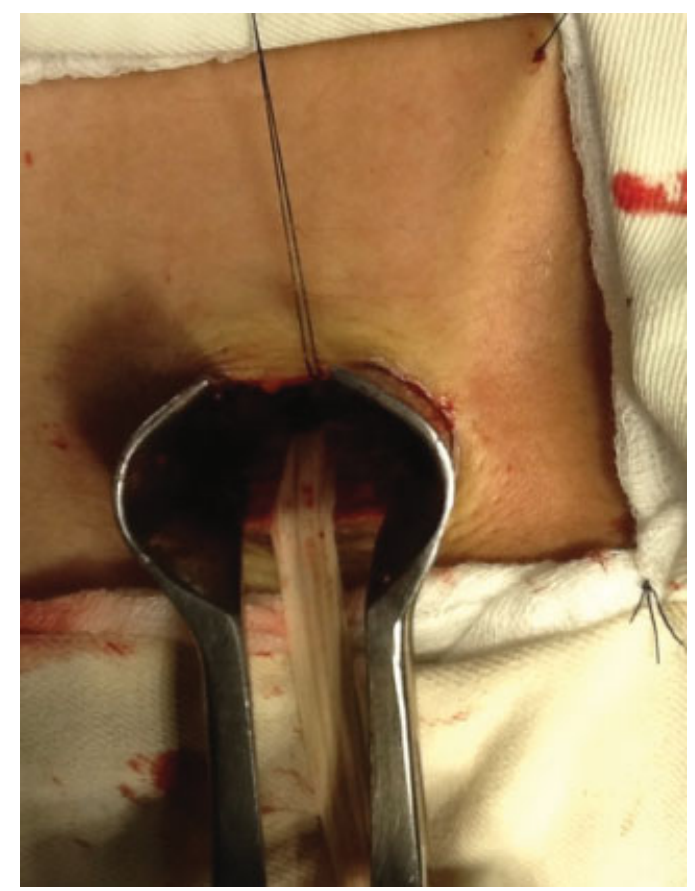

Fig. 12 Afastador de Caspar e dreno de Penrose. 


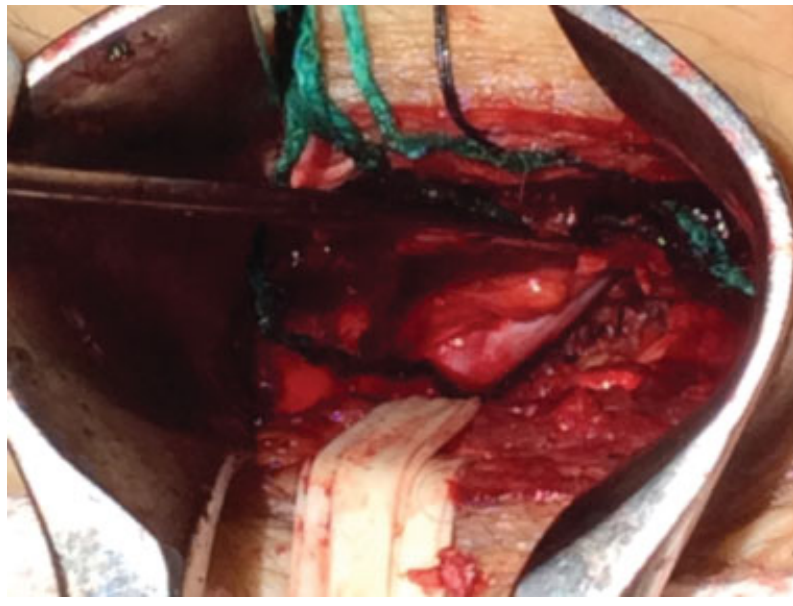

Fig. 13 Afastador de Caspar e dreno de Penrose com visualização macroscópica da raiz e do saco dural.

se um gancho de ponta romba e explora-se o ligamento amarelo no espaço epidural para liberá-lo de possíveis aderências à raiz ou ao saco dural.

Com o auxílio de Kerrison de $2 \mathrm{~mm}$, abre-se lateralmente o ligamento amarelo evitando usar lâmina de bisturi para não abrir a dura-máter do saco dural ou da bainha da raiz. Uma vez aberta a parte lateral do ligamento amarelo, rebate-se medialmente com ancoragem com fio de náilon de $2 \mathrm{~mm}$. Se a raiz não for visualizada, realiza-se a ligamentectomia lateral do restante do ligamento amarelo e pequena parte do recesso lateral. Então afasta-se a raiz e explora-se o disco herniado, sempre afastando a raiz pelo ombro; deve-se evitar retirar o disco sem visualização da raiz. Realiza-se incisão em cruz no disco herniado e faz-se a pulpectomia. Observa-se a mobilidade da raiz após a pulpectomia, explorando possíveis fragmentos migrados cranial, medial, lateral e caudalmente em direção ao forame. Irriga-se sob pressão com jato de soro fisiológico em seringa no espaço discal e aspiram-se restos de núcleo pulposo se existirem. Se a raiz não apresentar mobilidade distal, explorar bem o forame e, caso não exista fragmento de hérnia intraforaminal, realizar a foraminotomia parcial que acontece nas hipertrofias facetárias das estenoses de recesso lateral. Um indicativo de melhora dos sintomas e sinais do paciente é quando a raiz estiver totalmente livre de compressão e com boa mobilidade no sentido proximal e distal. Após isso, realiza-se hemostasia com Surgicel@ (Somerville, EUA) ou Gelfoam ${ }^{\circledR}$ (New York, EUA) ou mesmo com bipolar. Cobre-se a raiz e o saco dural com a gordura epidural pediculada se existente no espaço, faz-se embebição com $2 \mathrm{ml}$ de esteroide de depósito que não contenha álcool tipo acetado de metilprednisolona de $40 \mathrm{mg}$ e devolve-se o ligamento amarelo a sua posição, cobrindo o espaço epidural (-Fig. 14). O ligamento amarelo não será preservado somente nos casos de hipertrofia ligamentar. Coloca-se dreno de sucção de $3,2 \mathrm{~mm}$ por 24 horas para prevenir coleção sanguínea e possível fibrose futura. Realiza-se a síntese da fáscia toracolombar com náilon de $2 \mathrm{~mm}$ para evitar hérnia muscular e em seguida a síntese da pele

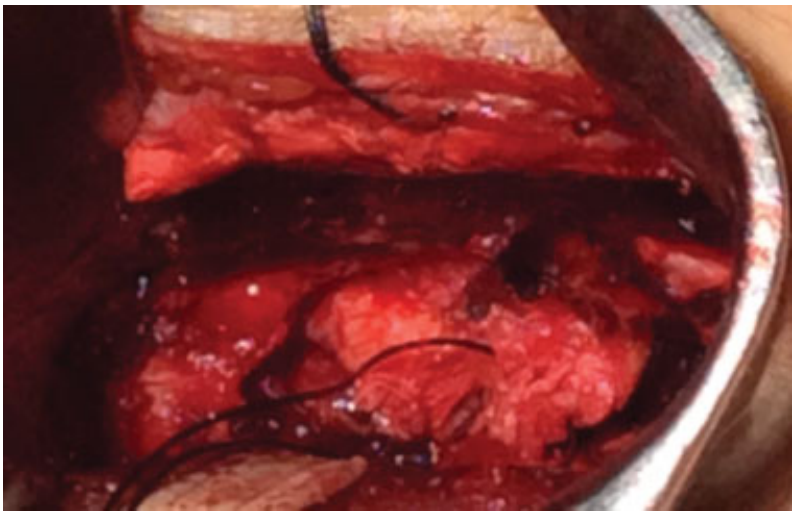

Fig. 14 Ligamento amarelo preservado.

(-Fig. 15). A alta hospitalar do paciente ocorre geralmente em 24 horas.

As - Figs. 16 a 19 representam cortes axiais de RNM de 2013 do paciente M.A.S., de 50 anos. Trata-se do controle pós-operatório de HDL L5-S1 esquerda submetida à microdiscectomia interlaminar em 1997. Observa-se mínima agressão às estruturas anatômicas.

É importante acrescentar que, com essa técnica, no acesso interlaminar podem ser abordados dois ou três espaços discais vizinhos com uma mesma incisão da pele. No caso

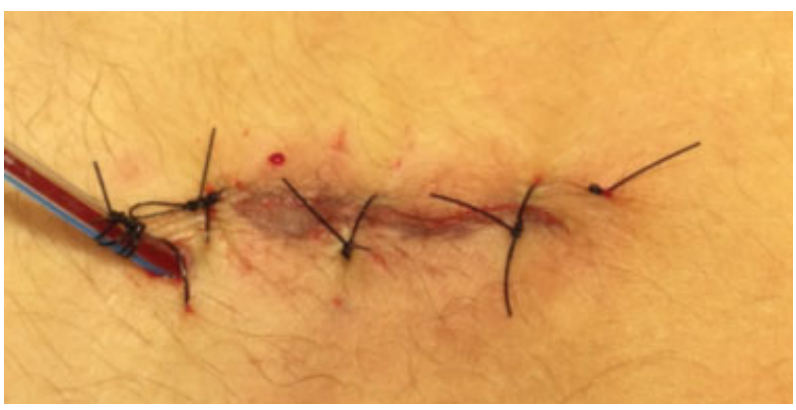

Fig. 15 Síntese da pele.

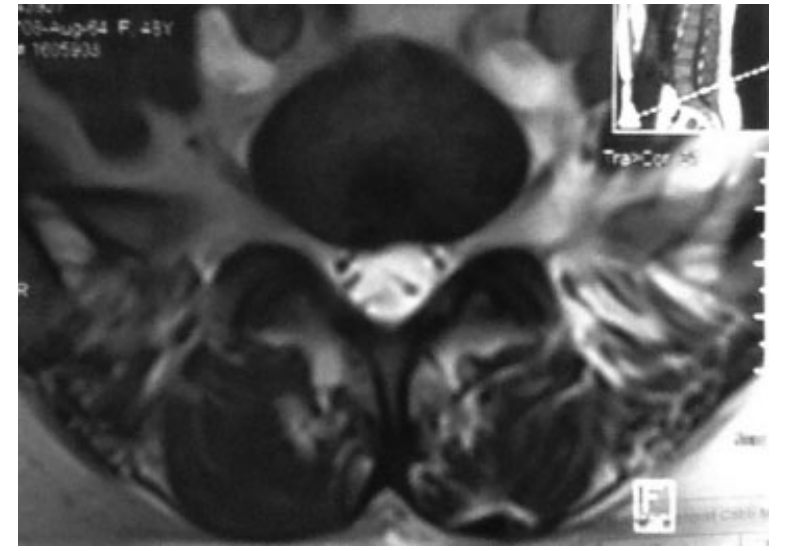

Fig. 16 Controle pós-operatório em 2013 de hérnia discal lombar L5S1 esquerda submetida à microdiscectomia interlaminar em 1997. 


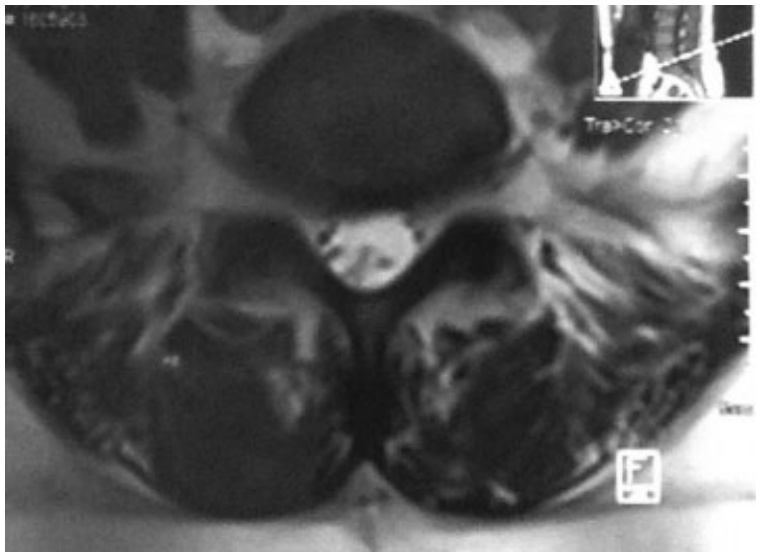

Fig. 17 Controle pós-operatório em 2013 de hérnia discal lombar L5S1 esquerda submetida à microdiscectomia interlaminar em 1997.

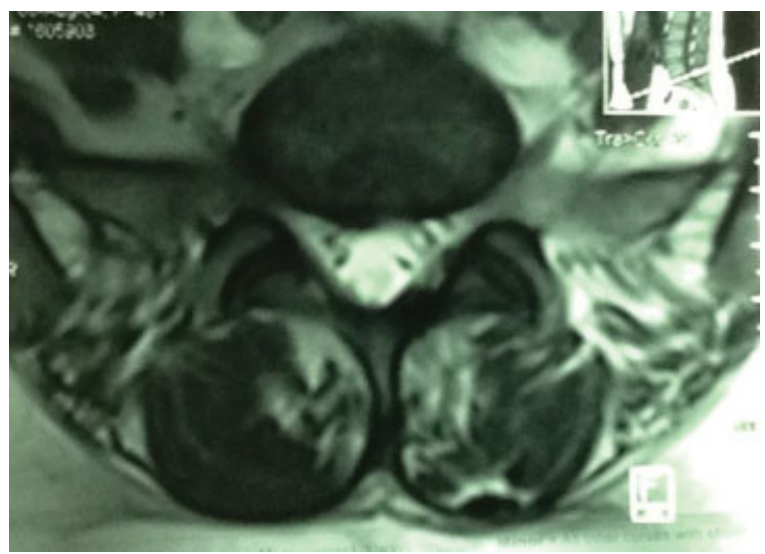

Fig. 18 Controle pós-operatório em 2013 de hérnia discal lombar L5S1 esquerda submetida à microdiscectomia interlaminar em 1997.

de dois espaços vizinhos, o ponto central da incisão da pele é o ponto médio entre os dois espaços discais. Se são três espaços vizinhos, o ponto central da incisão é o disco do meio. Existe maior facilidade ao abordar dois ou três espaços quando se coloca chumaço de três gazes amarrado em

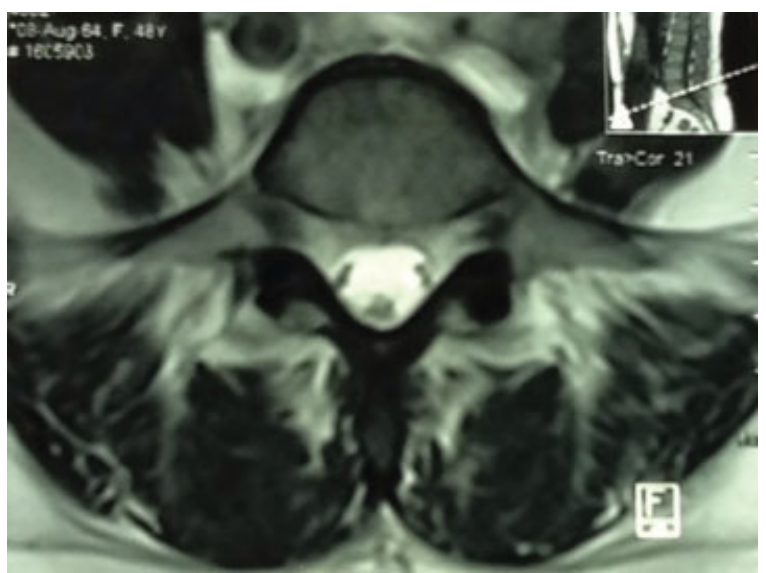

Fig. 19 Controle pós-operatório em 2013 de hérnia discal lombar L5S1 esquerda submetida à microdiscectomia interlaminar em 1997.

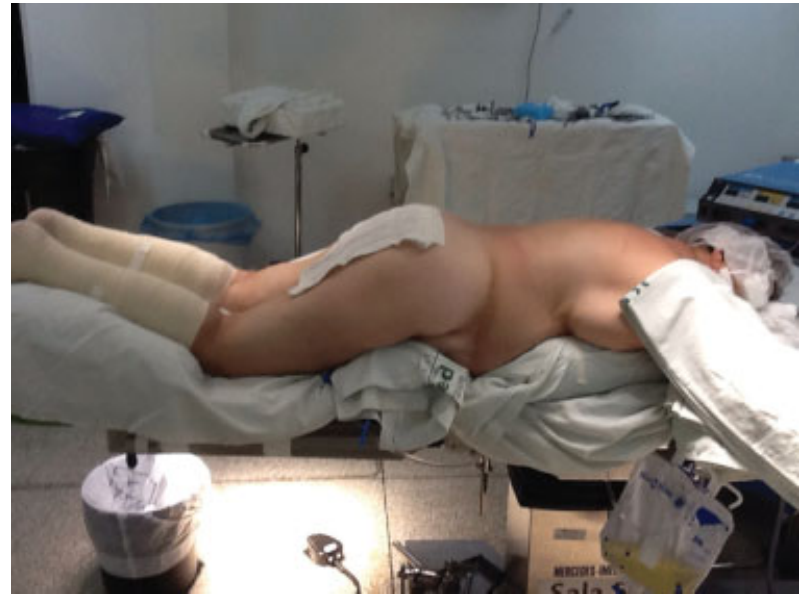

Fig. 20 Posição cirúrgica do acesso intertransverso.

Algofil $^{\mathrm{TM}}$ (São Paulo, Brasil). Coloca-se o chumaço de gaze entre a musculatura e a lâmina cranial do Caspar quando se deseja maior expansão da lâmina caudal. Quando se deseja maior expansão da lâmina cranial, o chumaço deve ser colocado entre a musculatura e a lâmina caudal. $O$ chumaço de gaze funciona como uma parede, facilitando a expansão da lâmina livre, ou seja, sem contato com o chumaço. Não se deve esquecer de retirar tal chumaço ao retirar o afastador de Caspar. O Algofil ${ }^{\mathrm{TM}}$ preso no chumaço também ajuda a não esquecer deste, pois o Algofil ${ }^{\mathrm{TM}}$ fica na pele.

\section{Acesso Intertransverso}

Neste acesso, operam-se as HDL extraforaminais, ou seja, as hérnias localizadas completamente fora do forame intervertebral ou pelo menos dois terços lateralmente ao pedículo. Na nossa cauística, operamos 12 hérnias extraforaminais $(2,39 \%)$. A posição cirúrgica é o decúbito ventral com membros inferiores semifletidos e com coxim em cintura pélvica e lateralmente ao tórax e ombros (-Fig. 20).

Realiza-se imagem lateral e posteroanterior com intensificador de imagem. Há como referências: medialmente, a pars interarticularis; superior e inferiormente, a apófise transversa superior e inferior, respectivamente; e lateralmente, a parte mais lateral das apófises transversas (-Fig. 21). A incisão é paramediana, em torno de $5 \mathrm{~cm}$ da linha média, e o acesso é intertransverso (-Fig. 22). Realizase a incisão na fáscia toracolombar em meia lua e rebate-se a fáscia medialmente. Afastam-se os feixes musculares do multifídio e longuíssimo, ou seja, via de Wiltse, ${ }^{16,17}$ usando dois afastadores tipo Langberg e coloca-se afastador de Caspar com uma inclinação de 10 a $15^{\circ}$ apoiando-o inferiormente nas apófises transversas. Como o afastador de Caspar é fixado na mesa cirúrgica com atadura de crepom, sua angulação pode ser diminuída ou aumentada lateral ou medialmente, cranial ou caudalmente a depender da necessidade. A mesa cirúrgica poderá ser inclinada no sentido superior (lateral para cima) para facilitar a visualização do campo com o microscópio. Um dreno de Penrose é colocado com auxílio de pinça Kelly curva na musculatura medial, e o mesmo procedimento é realizado na musculatura lateral. 


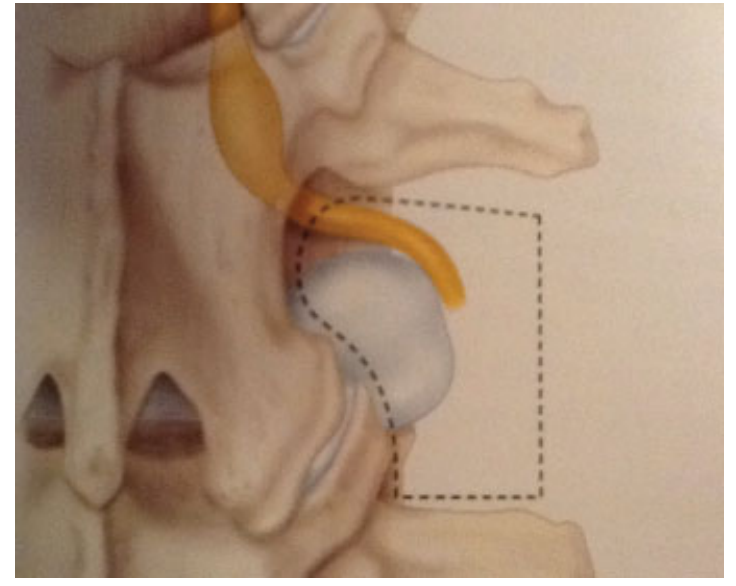

Fig. 21 Referências do acesso intertransverso. Fonte: Vaccaro e Baron $^{23}$

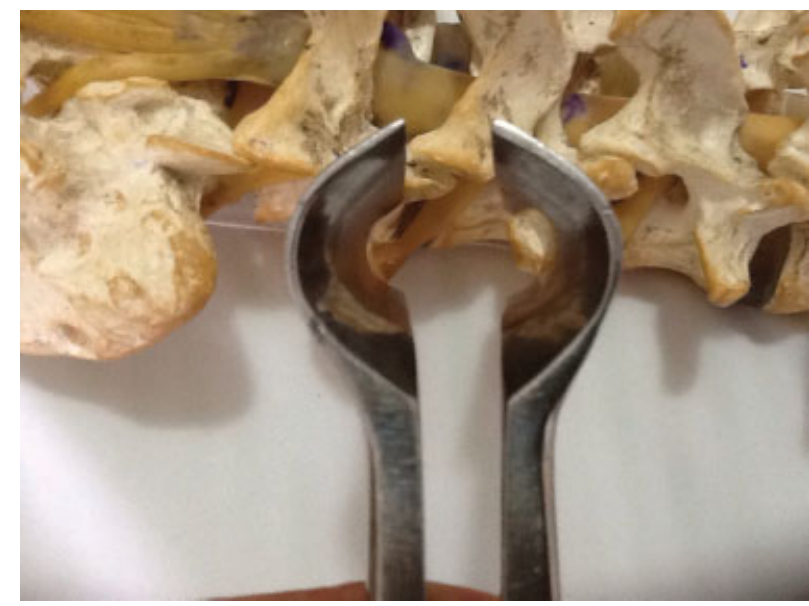

Fig. 22 Acesso intertransverso.

Com a tração dos drenos nos sentidos lateral e medial, existe excelente exposição do campo sem atrapalhar o trabalho do cirurgião, pois os drenos afastam a musculatura e praticamente não aparecem no campo. Coloca-se o microscópio cirúrgico, e a metade medial do músculo intertransverso é incisada e rebatida lateralmente para expor o ligamento ou membrana intertransversária. Localiza-se a raiz lateral (superior) e medial (inferior) em relação com o músculo psoas maior que estão embutidas na gordura extraforaminal e no tecido conjuntivo, por baixo da membrana intertransversária. A retração suave e cuidadosa da raiz superior em sentido superior e lateral permite melhor acesso ao material discal. Identificando o pedículo inferior e a dissecção proximal é possivel a identificação do disco. Os ramos da artéria lombar deverão ser dissecados e poupados. As veias podem ser tamponadas com Surgicel@ , Gelfoam ${ }^{\circledR}$ ou com bipolar, buscando-se evitar aquecimento das estruturas neurais. A retirada do material herniado deve ser realizada com pinça Love delicada de $2 \mathrm{~mm}$. Com gancho de ponta romba pesquisa-se fragmento discal no canal da raiz. Após a hemostasia adequada, é realizada a embebição das estruturas neurais com $2 \mathrm{ml}$ de acetato de metilpred- nisolona de $40 \mathrm{mg}$. Coloca-se dreno de sucção de 3,2 mm e procede-se com a síntese.

No nível L5-S1 geralmente há necessidade de remoção do processo transverso de L5 e desbaste lateral facetário. É importante lembrar que em pacientes com idade mais avancada, geralmente acima de 50 anos, poderá ocorrer um quadro de dor na região anterior da coxa, paresia quadricipital, reflexo patelar abolido e positividade do estiramento passivo do nervo femoral. Nesse caso, devese pensar em HDL extraforaminal, que corresponde a $10 \%$ das hérnias discais sintomáticas. ${ }^{18-21}$ Elas se apresentam em 75\% no nível de L4-L5 e acima, sendo que $46 \%$ ocorrem em L2-L3 e L3-L4. São geralmente mais dolorosas que as intraespinhais, em virtude da localização do GRD nas raízes lombares ser no FIL. ${ }^{22}$

\section{Conclusão}

O afastador de Taylor é o que oferece melhor afastamento lateral, porém apresenta o inconveniente de fratura ou degeneração de faceta articular, além de não oferecer um bom afastamento no sentido cranial e caudal. Os afastadores de Williams, Valin, Scoville e Markham-Meyerding oferecem bom afastamento lateral e pobre afastamento cranial e caudal. Os tubos dilatadores usados para endoscopia, por não serem retratores, exigem necessidade de troca por tubos de maior diâmetro para ter um campo operatório melhor e, como os acima citados, apresentam também o inconveniente de realizar duas ou três incisões na pele, caso seja necessário abordar dois ou três espaços discais vizinhos. $\mathrm{O}$ afastador de Caspar tem excelente afastamento cranial-caudal e pobre medial-lateral. A associação do afastador de Caspar tipo tubular cilíndrico com o dreno de Penrose de $30 \mathrm{~cm}$ de comprimento e $3 \mathrm{~cm}$ de largura oferece com uma pequena incisão de pele um excelente campo operatório no sentido lateral, medial, cranial e caudal, trabalho fácil com as duas mãos e mínima agressão às estruturas anatômicas, como mostram as - Figs. 16 a 19, sem perda funcional da musculatura e de baixo custo. Esta associação pode ser usada nos acessos interlaminar e intertransverso. Nos acessos interlaminares, ela pode ser usada em até três espaços discais vizinhos com a mesma incisão de pele.

\section{Conflito de Interesses}

Os autores declaram inexistência de conflito de interesse na realização deste trabalho.

\section{Referências}

1 Rothman RS, Simeone FA. La coluna vertebral. Buenos Aires: Panamerica; 1985:476-662

2 Santos JC, Lima PJC. Microdiscectomia lombar: técnica e resultados em 80 casos. Arq Neuropsiq 1993;51(2):205

3 Oppenheim H, Krause F. Uber Einklemmung bzw Strangulation der Cauda equina. Dtsch Med 1909;35:697-700

4 Mixter WS, Barr IS. Rupture of intervertebral disc without involvement of the spinal cord. N Engl J Med 1934;211:210-215 
5 Maroon JC, Abla AA. Microlumbar discectomy. Clin Neurosurg 1986;33:407-417

6 Love JG. Removal of protuded intervertebral disks without laminectomy. Proc Staff Meet Mayo Clin 1939;14:800

7 Goald HJ. Microlumbar discectomy: follow-up of 477 patients. J Microsurg 1980;2(2):95-100

8 Williams RW. Microlumbar discectomy: a conservative surgical approach to the virgin herniated lumbar disc. Spine 1978;3(2): 175-182

9 Ahn UM, Ahn NU, Buchowski JM, Garrett ES, Sieber AN, Kostuik JP. Cauda equina syndrome secondary to lumbar disc herniation: a meta-analysis of surgical outcomes. Spine 2000;25(12):1515-1522

10 Cox JM. Dor lombar. Chap 3. Cox JM. Neurofisiologia e patologia da raiz nervosa e do gânglio da raiz dorsal. 6th ed. São Paulo: Manole; 2002:131-132

11 Fardon DF, Milette PC. Combined Task Forces of the North American Spine Society, American Society of Spine Radiology, and American Society of Neuroradiology. Nomenclature and classification of lumbar disc pathology. Recommendations of the Combined task Forces of the North American Spine Society, American Society of Spine Radiology, and American Society of Neuroradiology. Spine 2001;26(5):E93-E113

12 Dantas FL, Fagundes-Pereyra WJ, Rocha DL, Raso JL. Giant cervical disc herniation: case report. Arq Neuropsiquiatr 1999;57 (2A):296-300

13 Williams RW. Microlumbar discectomy. A 12-year statistical review. Spine 1986;11(8):851-852

14 Hakelius A. Prognosis in sciatica. A clinical follow-up of surgical and non-surgical treatment. Acta Orthop Scand Suppl 1970; 129:1-76
15 Shapiro S. Cauda equina syndrome secondary to lumbar disc herniation. Neurosurgery 1993;32(5):743-746, discussion 746747

16 Wiltse LL, Bateman JG, Hutchinson RH, Nelson WE. The paraspinal sacrospinalis-splitting approach to the lumbar spine. J Bone Joint Surg Am 1968;50(5):919-926

17 Wiltse LL, Spencer CW. New uses and refinements of the paraspinal approach to the lumbar spine. Spine 1988;13(6): 696-706

18 Epstein NE. Evaluation of varied surgical approaches used in the management of 170 far-lateral lumbar disc herniations: indications and results. J Neurosurg 1995;83(4):648-656

19 Epstein NE, Epstein JA, Carras R, Hyman RA. Far lateral lumbar disc herniations and associated structural abnormalities. An evaluation in 60 patients of the comparative value of CT, MRI, and myelo-CT in diagnosis and management. Spine 1990;15(6): 534-539

20 Lanzino G, Shaffrey CI, Jane JA. Surgical treatment of lateral lumbar herniated discs. In: Rengachary SS, Wilkins RH, eds. Neurosurgical operativeatlas. Lebanon: American Assocation of Neurological Surgeons; 1999:243-251

21 Vroomen PC, de Krom MC, Wilmink JT. Pathoanatomy of clinical findings in patients with sciatica: a magnetic resonance imaging study. J Neurosurg 2000;92(2, Suppl):135-141

22 Ohmori K, Kanamori M, Kawaguchi Y, Ishihara H, Kimura T. Clinical features of extraforaminal lumbar disc herniation based on the radiographic location of the dorsal root ganglion. Spine 2001;26(6):662-666

23 Vaccaro AR, Baron EM, eds. Cirurgia da Coluna Vertebral. Rio de Janeiro: Elsevier; 2009 